\title{
A Spin-Perturbation for Minimal Surfaces
}

\section{Ruijun $\mathrm{Wu}^{1}$}

Received: 19 May 2020 / Accepted: 13 February 2021 / Published online: 14 May 2021

(C) The Author(s) 2021

\begin{abstract}
We investigate the coupling of the minimal surface equation with a spinor of harmonic type. This arises as the Euler-Lagrange equations of the sum of the volume functional and the Dirac action, defined on an appropriated Dirac bundle. The solutions show a relation to Dirac-harmonic maps under some constraints on the energy-momentum tensor, extending the relation between Riemannian minimal surface and harmonic maps.
\end{abstract}

Keywords SP-minimal surface $\cdot$ Dirac-harmonic maps · Energy-momentum tensor

Mathematics Subject Classification (2010) 58E12 · 49Q05 · 53C43 · 70H09

\section{Introduction}

Among many interesting and rich structures of the Dirac-harmonic maps introduced in [4], we are interested in those with special domain metrics. The properties of the domain metrics can be reflected by the energy-momentum tensor of the corresponding Lagrangian. Here we will see that, in special cases, the Dirac-harmonic maps admit another geometric interpretation, which is similar to minimal surface and will be called $S P$-minimal surfaces. This is the object we are going to study.

As a motivation, recall that the energy-momentum tensor for the Dirichlet energy along a harmonic map $u: M \rightarrow N$ from a Riemann surface $M$ gives rise to a holomorphic quadratic differential over $M$, which represents a cotangent vector in the Teichmüller space of $M[11,22]$. In particular, when the metric is critical with respect to variations, the energymomentum tensor vanishes and the map $u$ is weakly conformal ("weakly" means it can admit ramifications). But for a weakly conformal map $u$ from a Riemann surface, it is a harmonic map iff it is a branched minimal immersion. This key observation provides an effective way to obtain minimal surfaces via harmonic maps, see e.g. [8, 21].

Dedicated to Jürgen Jost.

Ruijun Wu

ruijun.wu@sissa.it

1 Scuola Internazionale Superiore di Studi Avanzati (SISSA), Via Bonomea, 265, 34136 Trieste, Italy 
The supersymmetric nonlinear sigma model gives rise to the fermionic analogue of the harmonic maps, namely we couple a map $u: M \rightarrow(N, h)$ with a spinor field $\psi$ along it and consider the action

$$
\int_{M}|\mathrm{~d} u|^{2}+\langle\not D \psi, \psi\rangle \mathrm{dvol}_{g}
$$

where $g$ is a fixed Riemannian metric on $M, \not D$ is the canonical Dirac operator defined for such spinors. The critical points of this action functional are known as Dirac-harmonic maps. They are reduced to harmonic maps if the spinor field $\psi$ is turned off. The energymomentum tensor of this functional is given by a symmetric 2-tensor $\mathcal{T}=\mathcal{T}_{i j} e^{i} \otimes e^{j}$ where $\left(e^{i}\right)$ is a local orthonormal coframe and

$$
\mathcal{T}_{i j}=2\left(u^{*} h\right)_{i j}-|\mathrm{d} u|^{2} g_{i j}+\frac{1}{2}\left\langle\gamma\left(e_{i}\right) \nabla_{e_{j}} \psi+\gamma\left(e_{j}\right) \nabla_{e_{i}} \psi, \psi\right\rangle-\langle\not D \psi, \psi\rangle g_{i j} .
$$

See [4] for more details.

Now it is natural to wonder to what the solutions would be reduced if the associated energy-momentum tensor of the Dirac-harmonic functional vanishes. This leads us to consider a perturbation of the volume functional of an immersion $u: M^{m} \rightarrow(\bar{M}, \bar{g})$ by a Dirac action:

$$
\mathcal{A}_{S}=\int_{M} m+\langle\not D \psi, \psi\rangle \operatorname{dvol}_{u^{*} \bar{g}},
$$

where $\psi$ is a spinor along the map $u$, see Section 2 . The critical points, which we might call "Dirac-minimal surfaces" at first glance, would be a good candidate. But we will save the name until it is formally judged; rather, let us be modest and call it a spin-perturbed minimal surface, or SP-minimal surface. The technical problem was the dependence of the spinor bundles on the pull-back metrics, but this can be handled with the well-known Bourguignon-Gauduchon approach [3, 13]. We will see that there do exist relations between the $S P$-minimal surfaces and the Dirac-harmonic maps similar to that between minimal surfaces and harmonic maps, provided that the energy-momentum tensor satisfies certain constraints. The vanishing of the energy-momentum tensor is sufficient but more than necessary. More precisely, our main result is the following

Theorem 1.1 Let $u: M^{m} \rightarrow(\bar{M}, \bar{g})$ be an isometric immersion from a Riemannian spin manifold and $\psi \in \Gamma\left(\Sigma_{g} M \otimes u^{*} T \bar{M}\right)$. Let $\mathcal{T}(u, \psi)$ be the energy-momentum tensor (1) for the Dirac-harmonic map functional.

1. Suppose $\mathcal{T}=(2-m) g$. Then $(u, \psi)$ is a Dirac-harmonic map iff $(u, \sqrt{m / 2} \psi)$ is an SP-minimal surface.

2. More generally, if $-\mathbb{I}(T)=2 \sigma \mathbf{H}$ for some $\sigma \geq 0$, then $(u, \psi)$ is a Dirac-harmonic map iff $(u, \sqrt{\sigma} \psi)$ is an SP-minimal surface, where $\mathbb{I}$ (resp. $\mathbf{H})$ are the second fundamental form (resp. mean curvature vector of the immersion), and $T$ is the energy-momentum tensor for (2) given by

$$
T=T_{i j} e^{i} \otimes e^{j}, \quad \text { with } T_{i j}=\frac{1}{2}\left\langle\gamma\left(e_{i}\right) \not_{e_{j}} \psi+\gamma\left(e_{j}\right) \not_{e_{i}} \psi, \psi\right\rangle-(m+\langle\not D \psi, \psi\rangle) g_{i j} .
$$

Here $\left(e_{i}\right)$ is a local orthonormal frame and $\left(e^{i}\right)$ is the dual coframe.

Note that in dimension two we may relax $u$ to be a conformal immersion, since the Dirac-harmonic functional is conformally invariant. Furthermore, the energy-momentum tensor for the Dirac-harmonic functional gives rise to a holomorphic quadratic differential along Dirac harmonic maps [4] in dimension two. However for a general supersymmetric 
nonlinear sigma model $[5,9,17]$ and for the Riemannian versions (e.g. a nonlinear sigma model with gravitino as in [12-14]), there are only conservation laws weaker than being holomorphic, except in some special cases as e.g. [16]. In this sense, the above generalized conditions for the energy-momentum tensor may be useful in understanding the associated quadratic differentials.

As another motivation, the volume of a super Riemann surface is not fully understood. The critical point there might be more appropriate to be called a Dirac-minimal surface. Note that the area or volume functional corresponds to the simplest Nambu-Goto invariant action in bosonic string theory [20]. The equation that we are going to study may only be a Riemannian simplification of the physics-relevant one. It is anyway interesting and heuristic to study our spin-perturbed area functional if we defend ourselves by the commuting and anti-commuting variables argument, which is well-known in the community. The main point here is to search for a reasonable geometric description of the Dirac-harmonic maps which may give rise to a moduli-type theory.

The paper is organized as follows. First we introduce the spin-perturbed volume functional whose critical points are the $S P$-minimal surfaces. Then we investigate the relations of such surfaces with Dirac-harmonic maps. In the end we also discuss some known examples.

When I was a student in Jürgen's group, he brought up the topic of supersymmetric variational problems. I had this question from him during the project on the nonlinear sigma models with gravitinos. This note is dedicated to him as a partial answer. I would like to take this chance to thank him for his constantly inspiring and encouraging discussions during the last years.

\section{The Spin-perturbed Volume Functional}

In this section we introduce the spin-perturbed volume functional and calculate the EulerLagrange equations for its critical points. Before turning to the perturbed case, we recall some basics of minimal surfaces in order to fix the notation. The spin-perturbed action functional shows some similarities to the Dirac-harmonic map functional, but note that the metric on $M$ varies along with the variation of the immersions, while in the harmonic maps and Dirac-harmonic maps setting the domain metrics are fixed. This difference has to be handled by introducing contractions of the energy-momentum tensor, as explained in the sequel.

\subsection{Review of the Minimal Surface Equation}

Here, we recall the first variation of the volume functional for an immersion and the variational field of the Riemannian metric.

Let $\left(\bar{M}^{m+m^{\prime}}, \bar{g}\right)$ be a Riemannian manifold and $u: M^{m} \rightarrow \bar{M}$ an immersion of codimension $m^{\prime} \geq 1$. With respect to the pull-back metric $g \equiv u^{*} \bar{g}$, the map $u$ is then an isometric immersion. The volume (or area) of $(M, g)$ is

$$
\mathcal{A}(u):=\int_{M} \mathrm{dvol}_{g}
$$

Consider a smooth variation $\left(u_{t}\right)$ of $u=u_{0}$, with variational field

$$
V:=\left.\frac{\partial u_{t}}{\partial t}\right|_{t=0} \in \Gamma\left(u^{*} T \bar{M}\right) \text {. }
$$


Assume that for each $t \in(-\varepsilon, \varepsilon)$, the map $u_{t}$ is a smooth immersion. This gives rise to a smooth variation $g(t) \equiv u_{t}^{*} \bar{g}$ of the metric $g=g(0)$.

Let $\left(e_{1}, \ldots, e_{m}, e_{m+1}, \ldots, e_{m+m^{\prime}}\right)$ be an oriented $\bar{g}$-orthonormal frame in a local neighborhood $U \subset \bar{M}$ such that $e_{i}, 1 \leq i \leq m$ are tangential to $u_{0}(M)$ and $e_{\alpha}, m+1 \leq \alpha \leq$ $m+m^{\prime}$ are normal to $u_{0}(M)$. We may further assume that the tangent frame $\left(e_{1}, \ldots, e_{m}\right)$ is geodesic at $x_{0}=u_{0}\left(x_{0}\right) \in M \cong u_{0}(M)$ with respect to $g=u_{0}^{*} \bar{g}$. Denoting the Levi-Civita connection of $g$ by $\nabla$, and that of $\bar{g}$ by $\bar{\nabla}$, then

$$
\nabla_{e_{i}} e_{j}\left(x_{0}\right)=\left(\bar{\nabla}_{e_{i}} e_{j}\right)^{\top}\left(x_{0}\right)=0 .
$$

This is equivalent to that the Christoffel symbols of $\bar{g}$ with tangential indices vanish: $\Gamma_{j k}^{i}\left(x_{0}\right)=0$. Similarly, we may assume that the normal frame $\left(e_{m+1}, \ldots, e_{m+m^{\prime}}\right)$ is chosen such that

$$
\left(\bar{\nabla}_{e_{i}} e_{\alpha}\right)^{\perp}\left(x_{0}\right)=0, \quad \text { or equivalently: } \quad \Gamma_{i \alpha}^{\beta}\left(x_{0}\right)=0 .
$$

We will use the Einstein summation convention wherever there is no confusion: any index appearing twice (not necessarily in upper and lower positions) is meant to be summed.

With respect to this frame,

$$
g(t)_{i j}=\left(u_{t}^{*} \bar{g}\right)_{i j}(x)=\bar{g}_{u_{t}(x)}\left(\mathrm{d} u_{t}\left(e_{i}\right), \mathrm{d} u_{t}\left(e_{j}\right)\right)
$$

and the linearization of the metric is

$$
\begin{aligned}
\left.\frac{\mathrm{d}}{\mathrm{d} t}\right|_{t=0} g(t)_{i j}(x)= & \left.\frac{\partial \bar{g}_{u_{t}(x)}}{\partial t}\left(e_{i}, e_{j}\right)\right|_{t=0}+\bar{g}_{u(x)}\left(\left.\frac{\mathrm{d}}{\mathrm{d} t}\right|_{t=0} \mathrm{~d} u_{t}\left(e_{i}\right), \mathrm{d} u_{t}\left(e_{j}\right)\right) \\
& +\bar{g}_{u(x)}\left(\mathrm{d} u_{t}\left(e_{i}\right),\left.\frac{\mathrm{d}}{\mathrm{d} t}\right|_{t=0} \mathrm{~d} u_{t}\left(e_{j}\right)\right),
\end{aligned}
$$

which is a symmetric 2 -tensor. Since $\bar{g}$ is $\bar{\nabla}$-parallel, we have

$$
\left.\frac{\mathrm{d}}{\mathrm{d} t}\right|_{t=0} g(t)_{i j}(x)=\bar{g}_{p}\left(\bar{\nabla}_{e_{i}} V, e_{j}\right)+\bar{g}_{p}\left(e_{i}, \bar{\nabla}_{e_{j}} V\right),
$$

where we have used $\left[e_{i}, V\right]=\left[\mathrm{d} u_{t}\left(e_{i}\right), \mathrm{d} u_{t}\left(\partial_{t}\right)\right]=0$ and hence $\bar{\nabla}_{V} e_{i}=\bar{\nabla}_{e_{i}} V$. Write $V=$ $V^{l} e_{l}+V^{\alpha} e_{\alpha}=V^{\top}+V^{\perp}$, then at $x_{0}$,

$$
\bar{\nabla}_{e_{i}} V=e_{i}\left(V^{l}\right) e_{l}+V^{l} \bar{\nabla}_{e_{i}} e_{l}+e_{i}\left(V^{\alpha}\right) e_{\alpha}+V^{\alpha} \bar{\nabla}_{e_{i}} e_{\alpha}
$$

Thus

$$
\begin{aligned}
\left.\frac{\mathrm{d}}{\mathrm{d} t}\right|_{t=0} g(t)_{i j}(x)= & e_{i}\left(V^{j}\right)+V^{l}\left\langle\bar{\nabla}_{e_{i}} e_{l}, e_{j}\right\rangle+V^{\alpha}\left\langle\bar{\nabla}_{e_{i}} e_{\alpha}, e_{j}\right\rangle \\
& +e_{j}\left(V^{i}\right)+V^{l}\left\langle\bar{\nabla}_{e_{j}} e_{l}, e_{i}\right\rangle+V^{\alpha}\left\langle\bar{\nabla}_{e_{j}} e_{\alpha}, e_{i}\right\rangle \\
= & \left(e_{i}\left(V^{j}\right)+e_{j}\left(V^{i}\right)\right)-2 V^{\alpha} \mathbb{I}_{i j}^{\alpha} \equiv k_{i j},
\end{aligned}
$$

where $\mathbb{I}_{i j}^{\alpha}$ are the coefficients of the second fundamental form $\mathbb{I}$ :

$$
\mathbb{I}_{i j}^{\alpha}=\left\langle\bar{\nabla}_{e_{i}} e_{j}, e_{\alpha}\right\rangle, \quad 1 \leq i, j \leq m, \quad m+1 \leq \alpha \leq m+m^{\prime} .
$$

Thus the variational field of $g(t)$ is given by $k=k_{i j} e^{i} \otimes e^{j} \in \operatorname{Sym}^{2}\left(T^{*} M\right)$ with

$$
k_{i j}=e_{i}\left(V^{j}\right)+e_{j}\left(V^{i}\right)-2 V^{\alpha} \mathbb{I}_{i j}^{\alpha}, \quad 1 \leq i, j \leq m .
$$


Let $\mathbf{H}:=\operatorname{tr}(\mathbb{I})$ be the mean curvature vector. ${ }^{1}$ It follows that

$$
\left.\operatorname{tr}(\dot{g}(0)) \equiv \frac{\mathrm{d}}{\mathrm{d} t}\right|_{t=0} g(t)_{i i}(x)=2 \operatorname{div}_{g}\left(V^{\top}\right)-2\left\langle V^{\perp}, \mathbf{H}\right\rangle .
$$

Now let $\left(e^{i}\right)$ be the dual frame for the cotangent bundle of $M$. The volume form for the metric $g(t)$ can be locally expressed as

$$
\operatorname{dvol}_{g(t)}=\sqrt{\operatorname{det}(g(t))} e^{1} \wedge \cdots \wedge e^{m} .
$$

Recall that

$$
\begin{aligned}
\frac{\mathrm{d}}{\mathrm{d} t} \sqrt{\operatorname{det}(g(t))} & =\frac{1}{2} \frac{1}{\sqrt{\operatorname{det}(g(t))}} \frac{\mathrm{d}}{\mathrm{d} t} \operatorname{det}(g(t))=\frac{1}{2} \frac{1}{\sqrt{\operatorname{det}(g(t))}} \operatorname{det}(g(t)) \operatorname{tr}\left(g(t)^{-1} \frac{\mathrm{d}}{\mathrm{d} t} g(t)\right) \\
& =\frac{1}{2} \sqrt{\operatorname{det}(g(t))} \operatorname{tr}\left(g(t)^{-1} \frac{\mathrm{d}}{\mathrm{d} t} g(t)\right) .
\end{aligned}
$$

At $t=0$, we have $g(0)_{i j}=\delta_{i j}$, hence

$$
\left.\frac{\mathrm{d}}{\mathrm{d} t}\right|_{t=0} \sqrt{\operatorname{det}(g(t))}=\frac{1}{2} \operatorname{tr}(\dot{g}(0))=\operatorname{div}_{g}\left(V^{\top}\right)-\left\langle V^{\perp}, \mathbf{H}\right\rangle .
$$

Therefore, the first variation formula for the volume functional is given by

$$
\left.\frac{\mathrm{d}}{\mathrm{d} t}\right|_{t=0} \mathcal{A}\left(u_{t}\right)=\int_{M}-\left\langle V^{\perp}, \mathbf{H}\right\rangle+\operatorname{div}_{g}\left(V^{\top}\right) \operatorname{dvol}_{g} .
$$

In the case where $M$ is either closed or complete and $V$ has compact support, then

$$
\left.\frac{\mathrm{d}}{\mathrm{d} t}\right|_{t=0} \mathcal{A}\left(u_{t}\right)=\int_{M}-\left\langle V^{\perp}, \mathbf{H}\right\rangle \mathrm{dvol}_{g} .
$$

Therefore $u$ is a critical point of $\mathcal{A}$ iff $\mathbf{H}=0$, i.e. $u$ is minimal.

\subsection{Spin-perturbation of the Volume Functional}

Now we are going to consider the coupling with a spinor field. From now on assume that $(M, g)$ is spin, with a fixed spin structure and with associated spinor bundle $\Sigma_{g} M$, where $g=u^{*} \bar{g}$. It has a canonical spin connection $\nabla^{s}=\nabla^{\Sigma M}$ and a Riemannian inner product $g^{s}$, together with a compatible Clifford map $\gamma: T M \rightarrow \operatorname{End}\left(\Sigma_{g} M\right)$ which satisfies the following Clifford relation

$$
\gamma(X) \gamma(Y)+\gamma(Y) \gamma(X)=-2 g(X, Y) \mathrm{Id}, \quad \forall X, Y \in \Gamma(T M) .
$$

Then the spin Dirac operator $\not \partial$ is canonically defined as $\not \partial=\gamma\left(e_{i}\right) \nabla_{e_{i}}^{s} \varphi$, for any spinor $\varphi \in$ $\Gamma\left(\Sigma_{g} M\right)$. For more material on spin geometry we refer to $[7,10,18]$.

For the family of metrics $g(t)$, the associated spinor bundles are isomorphic, and there are isometries

$$
b_{t}:(T M, g(0)) \rightarrow(T M, g(t)) \quad \text { and } \quad \beta_{t}: \Sigma_{g(0)} M \rightarrow \Sigma_{g(t)} M
$$

with respect to the corresponding inner products on the bundles $[13,23]$ and

$$
\left.\frac{\mathrm{d}}{\mathrm{d} t}\right|_{t=0} b_{t}=-\frac{1}{2} K \in \operatorname{End}(T M),
$$

\footnotetext{
${ }^{1}$ In other convention (e.g. [1]) the mean curvature vector may be defined as $m^{-1} \operatorname{tr}(\mathbb{I})$, which differs from ours by a dimension factor. We use this convention to be consistent with the tension field for harmonic maps, see the beginning of Section 3 .
} 
where $k$ is the variational field of the metric and $K$ the associated endomorphism on $T M$. Moreover, pulling back the spin Dirac operator $\not_{g(t)}: \Gamma\left(\Sigma_{g(t)} M\right) \rightarrow \Gamma\left(\Sigma_{g(t)} M\right)$ via the above isometry $\beta_{t}$, we obtain a differential operator

$$
\overline{\not ̆}_{t}:=\beta_{t}^{-1} \circ \not_{g(t)} \circ \beta_{t}: \Gamma\left(\Sigma_{g(0)} M\right) \rightarrow \Gamma\left(\Sigma_{g(0)} M\right) .
$$

The linearization of $\bar{\not}_{t}$ was computed in [3, Theorem 21] (see also [19, Section 2])

$$
\left.\frac{\mathrm{d}}{\mathrm{d} t}\right|_{t=0} \overline{\not ̆}_{t}=-\frac{1}{2} \gamma\left(e_{i}\right) \nabla_{K\left(e_{i}\right)}^{s}+\frac{1}{4}\left(\operatorname{grad}_{g}\left(\operatorname{tr}_{g}(k)\right)-\operatorname{div}_{g}(k)_{\sharp}\right),
$$

where the divergence $\operatorname{div}_{g}$ on symmetric 2-tensors is as in $[13,22]$ and $\sharp: T^{*} M \rightarrow T M$ denotes the musical isomorphism.

Given an isometric immersion $u: M \rightarrow \bar{M}$, consider the bundle ${ }^{2} \Sigma_{g} M \otimes u^{*} T \bar{M}$. The connection, inner product and Clifford multiplication are naturally extended on this product bundle, denoted by $\not \mid \nabla^{\Sigma_{g} M \otimes u^{*} T \bar{M}}, g^{s} \otimes \bar{g}$ and ${ }^{3} \gamma$ respectively. Thus it becomes a Dirac bundle in the sense of [18, Def. 5.2] and we have as well the Dirac operator

$$
\not D=\gamma\left(e_{i}\right) \not_{e_{i}},
$$

which is a formally self-adjoint, first order elliptic differential operator. In terms of the local orthonormal frame $\left(e_{a}\right)$, we can write a spinor $\psi \in \Gamma\left(\Sigma_{g} M \otimes u^{*} T \bar{M}\right)$ as

$$
\psi=\psi^{a} \otimes e_{a}=\psi^{i} \otimes e_{i}+\psi^{\alpha} \otimes e_{\alpha},
$$

where the index $a$ runs through 1 to $m+m^{\prime}$. Then the Dirac operator acts as

$$
\not D \psi=\not \partial \psi^{a} \otimes e_{a}+\gamma\left(e_{i}\right) \psi^{a} \otimes \bar{\nabla}_{e_{i}} e_{a},
$$

or, in terms of the tangent and normal frames,

$$
\begin{aligned}
\not D \psi & =\not \partial \psi^{j} \otimes e_{j}+\gamma\left(e_{i}\right) \psi^{j} \otimes \bar{\nabla}_{e_{i}} e_{j}+\not \partial \psi^{\alpha} \otimes e_{\alpha}+\gamma\left(e_{i}\right) \psi^{\alpha} \otimes \bar{\nabla}_{e_{i}} e_{\alpha} \\
& =\not \partial \psi^{j} \otimes e_{j}+\gamma\left(e_{i}\right) \psi^{j} \otimes \mathbb{I}_{i j}^{\alpha} e_{\alpha}+\not \partial \psi^{\alpha} \otimes e_{\alpha}-\gamma\left(e_{i}\right) \psi^{\alpha} \otimes \mathbb{I}_{i j}^{\alpha} e_{j}, \quad \text { at } x_{0} .
\end{aligned}
$$

The spin-perturbation of the volume functional that we are going to consider has the form

$$
\mathcal{A}_{S}(u, \psi)=\int_{M} m+\langle\not D \psi, \psi\rangle \operatorname{dvol}_{u^{*} g} .
$$

Here $m=\operatorname{dim} M$ and if $u$ is an isometric immersion then $|\mathrm{d} u|^{2}=m$. In contrast to the Dirac-harmonic map functional in [4], there is no conformal symmetry for $\mathcal{A}_{S}$, because of the presence of the Riemannian volume part. Nevertheless it is invariant under diffeomorphisms: for $f \in \operatorname{Diff}(M)$ which preserves the orientation and the spin structure, we have

$$
\mathcal{A}_{S}\left(f^{*} u, f^{*} \psi\right)=\mathcal{A}_{S}(u, \psi)
$$

\subsection{The Variation Formulas}

Here, we derive the Euler-Lagrange equations for (6). The calculation is similar to that in [12]. We remark that there is an alternative method adopted in [2] which gives another viewpoint of such problems.

Let $(u, \psi)$ be a critical point and let $\left(u_{t}, \psi_{t}\right)_{-\varepsilon<t<\varepsilon}$ be a variation of $(u, \psi)$, where $u_{0}=u, \psi_{0}=\psi$, and for each $t \in(-\varepsilon, \varepsilon), u_{t}$ is an immersion, and $\psi_{t} \in \Gamma\left(\Sigma_{g(t)} M \otimes\right.$

\footnotetext{
${ }^{2}$ We always take tensor products over $\mathbb{R}$.

${ }^{3}$ We use the same notation for the Clifford map as that for $\Sigma_{g} M$ since they are essentially the same.
} 
$\left.u_{t}^{*} T \bar{M}\right)$. As we have seen the variation of the volume of $(M, g(t))$, it remains to compute the variation of the Dirac action:

$\left.\frac{\mathrm{d}}{\mathrm{d} t}\right|_{t=0} \int_{M}\left\langle\not D_{t} \psi_{t}, \psi_{t}\right\rangle \operatorname{dvol}_{g(t)}=\left.\int_{M} \frac{\partial}{\partial t}\right|_{t=0}\left\langle\not D_{t} \psi_{t}, \psi_{t}\right\rangle \operatorname{dvol}_{g}+\left.\int_{M}\langle\not D \psi, \psi\rangle \frac{\partial}{\partial t}\right|_{t=0} \operatorname{dvol}_{g(t)}$.

We need to compute the first term in the RHS. Note that $e_{i}(t)=b_{t}\left(e_{i}\right)$ are $g(t)$-orthonormal, and the $b_{t}$ can be extended to the normal bundle such that $e_{\alpha}(t)=b_{t}\left(e_{\alpha}\right)$ form an orthonormal frame along $u_{t}$ for the normal bundle. Also note that $\gamma_{t}\left(b_{t}\left(e_{i}\right)\right)$ can be taken to be $t$-independent. In terms of (4) and (5), noting that $\left(e_{a}(t)\right)$ are orthonormal, we have

$$
\begin{aligned}
\left\langle\not D_{g(t)} \psi_{t}, \psi_{t}\right\rangle_{g^{s}(t)}= & \left\langle\partial_{g(t)} \psi_{t}^{a} \otimes e_{a}(t)+\gamma_{t}\left(e_{i}(t)\right) \psi_{t}^{a} \otimes \bar{\nabla}_{e_{i}(t)} e_{a}(t), \psi_{t}^{b} \otimes e_{b}(t)\right\rangle \\
= & \left\langle\partial_{g(t)} \psi_{t}^{a}, \psi_{t}^{b}\right\rangle_{g^{s}(t)} \delta_{a b}+\left\langle\gamma_{t}\left(e_{i}(t)\right) \psi_{t}^{a}, \psi_{t}^{b}\right\rangle_{g^{s}(t)}\left\langle\bar{\nabla}_{e_{i}(t)} e_{a}(t), e_{b}(t)\right\rangle_{\bar{g}} \\
= & \left\langle\beta_{t}^{-1} \not_{g(t)} \beta_{t} \circ \beta_{t}^{-1} \psi_{t}^{a}, \beta_{t}^{-1} \psi_{t}^{b}\right\rangle_{g^{s}} \delta_{a b} \\
& +\left\langle\beta_{t}^{-1} \gamma_{t}\left(e_{i}(t)\right) \psi_{t}^{a}, \beta_{t}^{-1} \psi_{t}^{b}\right\rangle_{g^{s}}\left\langle\bar{\nabla}_{e_{i}(t)} e_{a}(t), e_{b}(t)\right\rangle_{\bar{g}} \\
= & \left\langle\bar{\partial}_{g(t)} \beta_{t}^{-1} \psi_{t}^{a}, \beta_{t}^{-1} \psi_{t}^{b}\right\rangle_{g^{s}} \delta_{a b} \\
& +\left\langle\gamma\left(e_{i}\right) \beta_{t}^{-1} \psi_{t}^{a}, \beta_{t}^{-1} \psi_{t}^{b}\right\rangle_{g^{s}}\left\langle\bar{\nabla}_{e_{i}(t)} e_{a}(t), e_{b}(t)\right\rangle_{\bar{g}} .
\end{aligned}
$$

Writing $\beta_{t}^{-1} \psi_{t}^{a} \equiv \phi_{t}^{a}$, the variational field of $\psi$ is given by

$$
\eta:=\left.\frac{\mathrm{d}}{\mathrm{d} t}\right|_{t=0} \phi_{t}^{a} \otimes e_{a}(t) \in \Gamma\left(\Sigma_{g} M \otimes u^{*} T \bar{M}\right) .
$$

Then we take the $t$-derivatives at $t=0$ :

$$
\begin{aligned}
& \left.\frac{\mathrm{d}}{\mathrm{d} t}\right|_{t=0}\left\langle\not D_{g(t)} \psi_{t}, \psi_{t}\right\rangle_{g^{s}(t)} \\
= & \left.\frac{\mathrm{d}}{\mathrm{d} t}\right|_{t=0}\left\langle\overline{\not \partial}_{g(t)} \phi_{t}^{a}, \phi_{t}^{b}\right\rangle_{g^{s}} \delta_{a b}+\left\langle\gamma\left(e_{i}\right) \phi_{t}^{a}, \phi_{t}^{b}\right\rangle_{g^{s}}\left\langle\bar{\nabla}_{e_{i}(t)} e_{a}(t), e_{b}(t)\right\rangle_{\bar{g}} \\
= & \left\langle\left(\left.\frac{\mathrm{d}}{\mathrm{d} t}\right|_{t=0} \bar{\not}_{g(t)}\right) \psi^{a}, \psi^{b}\right\rangle \delta_{a b}+\left\langle\not \partial_{g}\left(\left.\frac{\mathrm{d}}{\mathrm{d} t}\right|_{t=0} \phi_{t}^{a}\right), \psi^{b}\right\rangle \delta_{a b}+\left\langle\partial_{g} \psi^{a},\left.\frac{\mathrm{d}}{\mathrm{d} t}\right|_{t=0} \phi_{t}^{b}\right\rangle \delta_{a b} \\
& +\left\langle\gamma\left(e_{i}\right)\left(\left.\frac{\mathrm{d}}{\mathrm{d} t}\right|_{t=0} \phi_{t}^{a}\right), \psi^{b}\right\rangle\left\langle\bar{\nabla}_{e_{i}} e_{a}, e_{b}\right\rangle+\left\langle\gamma\left(e_{i}\right) \psi^{a},\left.\frac{\mathrm{d}}{\mathrm{d} t}\right|_{t=0} \phi_{t}^{b}\right\rangle\left\langle\bar{\nabla}_{e_{i}} e_{a}, e_{b}\right\rangle \\
& +\left\langle\gamma\left(e_{i}\right) \psi^{a}, \psi^{b}\right\rangle\left\langle\left(\partial_{t} \bar{\nabla}_{e_{i}(t)} e_{a}(t)\right)_{t=0}, e_{b}\right\rangle+\left\langle\gamma\left(e_{i}\right) \psi^{a}, \psi^{b}\right\rangle\left\langle\bar{\nabla}_{e_{i}} e_{a},\left.\frac{\mathrm{d}}{\mathrm{d} t}\right|_{t=0} e_{b}(t)\right\rangle .
\end{aligned}
$$

For the first term, we use (3) and the skewness of the Clifford multiplication to get

$$
\left\langle\left(\left.\frac{\mathrm{d}}{\mathrm{d} t}\right|_{t=0} \overline{\not \partial}_{g(t)}\right) \psi^{a}, \psi^{b}\right\rangle \delta_{a b}=-\frac{1}{2}\left\langle\gamma\left(e_{i}\right) \nabla_{K\left(e_{i}\right)}^{s} \psi^{a}, \psi^{b}\right\rangle \delta_{a b} .
$$

Viewing the $t$-derivatives as a covariant derivative on the product manifold $\mathbb{R} \times \bar{M}$ whose connection will still be denoted by $\bar{\nabla}$ (see e.g. [12] and also compare with [2]), we have

$$
\partial_{t} \bar{\nabla}_{e_{i}(t)} e_{a}(t)=\bar{R}\left(\mathrm{~d} u_{t}\left(\partial_{t}\right), e_{i}(t)\right) e_{a}(t)+\bar{\nabla}_{e_{i}(t)}\left(\bar{\nabla}_{\partial_{t}} e_{a}(t)\right)+\bar{\nabla}_{\left[\partial_{t}, e_{i}(t)\right]} e_{a}(t) .
$$

At $t=0$, this is

$$
\left(\partial_{t} \bar{\nabla}_{e_{i}(t)} e_{a}(t)\right)_{t=0}=\bar{R}\left(V, e_{i}\right) e_{a}+\bar{\nabla}_{e_{i}(t)}\left(\bar{\nabla}_{\partial_{t}} e_{a}(t)\right)_{t=0}-\frac{1}{2} \bar{\nabla}_{K\left(e_{i}\right)} e_{a}
$$


Note that

$$
0=\left.\frac{\mathrm{d}}{\mathrm{d} t}\right|_{t=0} \delta_{a b}=\left\langle\left.\frac{\mathrm{d}}{\mathrm{d} t}\right|_{t=0} e_{a}(t), e_{b}\right\rangle+\left\langle e_{a},\left.\frac{\mathrm{d}}{\mathrm{d} t}\right|_{t=0} e_{b}\right\rangle .
$$

Plugging these into the $t$-derivative of $\langle\not D \psi, \psi\rangle$ and evaluating at $t=0$, we get

$$
\begin{aligned}
\left.\frac{\mathrm{d}}{\mathrm{d} t}\right|_{t=0}\left\langle\not D_{g(t)} \psi_{t}, \psi_{t}\right\rangle_{g^{s}(t)}= & -\frac{1}{2}\left\langle\gamma\left(e_{i}\right) \nabla_{K\left(e_{i}\right)}^{s} \psi^{a}, \psi^{b}\right\rangle \delta_{a b}-\frac{1}{2}\left\langle\gamma\left(e_{i}\right) \psi^{a}, \psi^{b}\right\rangle\left\langle\bar{\nabla}_{K\left(e_{i}\right)} e_{a}, e_{b}\right\rangle \\
& +\left\langle\not D\left(\left.\frac{\mathrm{d}}{\mathrm{d} t}\right|_{t=0} \phi_{t}^{a} \otimes e_{a}(t)\right), \psi\right\rangle+\left\langle\not D \psi,\left(\left.\frac{\mathrm{d}}{\mathrm{d} t}\right|_{t=0} \phi_{t}^{b} \otimes e_{b}(t)\right)\right\rangle \\
& +\left\langle\gamma\left(e_{i}\right) \psi^{a}, \psi^{b}\right\rangle\left\langle\bar{R}\left(V, e_{i}\right) e_{a}, e_{b}\right\rangle \\
= & -\frac{1}{2}\left\langle\gamma\left(e_{i}\right) \not_{K\left(e_{i}\right)} \psi, \psi\right\rangle+\langle\not D \eta, \psi\rangle+\langle\not D \psi, \eta\rangle+\langle\mathscr{R}, V\rangle,
\end{aligned}
$$

where we have used the abbreviation

$$
\mathscr{R} \equiv\left\langle\psi^{a}, \gamma\left(e_{i}\right) \psi^{b}\right\rangle \bar{R}\left(e_{a}, e_{b}\right) e_{i}
$$

so that

$$
\left\langle\gamma\left(e_{i}\right) \psi^{a}, \psi^{b}\right\rangle\left\langle\bar{R}\left(V, e_{i}\right) e_{a}, e_{b}\right\rangle=\langle\mathscr{R}, V\rangle .
$$

Define a symmetric 2-tensor $T=T_{i j} e^{i} \otimes e^{j} \in \operatorname{Sym}^{2}\left(T^{*} M\right)$ by

$$
T_{i j}=\frac{1}{2}\left\langle\gamma\left(e_{i}\right) \not_{e_{j}} \psi+\gamma\left(e_{j}\right) \not_{e_{i}} \psi, \psi\right\rangle-(m+\langle\not D \psi, \psi\rangle) g_{i j}, \quad 1 \leq i, j \leq m .
$$

This is the energy-momentum tensor for the functional (6). Then

$$
\begin{aligned}
\left\langle\gamma\left(e_{i}\right) \not_{K\left(e_{i}\right)} \psi, \psi\right\rangle= & \langle k, T+(m+\langle\not D \psi, \psi\rangle) g\rangle \\
= & \left(e_{i}\left(V^{j}\right)+e_{j}\left(V^{i}\right)-2 V^{\alpha} \mathbb{I}_{i j}^{\alpha}\right) T_{i j}+(m+\langle\not D \psi, \psi\rangle) \operatorname{tr}_{g}(k) \\
= & 2\left(e_{j}\left(T_{i j} V^{i}\right)-V^{i} e_{j}\left(T_{i j}\right)\right)-2 V^{\alpha} \mathbb{I}_{i j}^{\alpha} T_{i j} \\
& +2(m+\langle\not D \psi, \psi\rangle)\left(\operatorname{div}_{g}\left(V^{\top}\right)-\left\langle V^{\perp}, \mathbf{H}\right\rangle\right) .
\end{aligned}
$$

Therefore,

$$
\begin{aligned}
\int_{M}\left\{\left.\frac{\mathrm{d}}{\mathrm{d} t}\right|_{t=0}\left\langle\not D_{t} \psi_{t}, \psi_{t}\right\rangle\right\} \mathrm{dvol}_{g}= & \int_{M} 2\langle\not D \psi, \eta\rangle-e_{i}\left\langle\eta, \gamma\left(e_{i}\right) \psi\right\rangle+\langle\mathscr{R}, V\rangle \mathrm{dvol}_{g} \\
& -\int_{M} \operatorname{div}_{g}\left(T\left(V^{\top}\right)\right)-\left\langle V^{\top}, \operatorname{div}_{g}(T)_{\sharp}\right\rangle-\left\langle V^{\perp}, \mathbb{I}(T)\right\rangle \operatorname{dvol}_{g} \\
& -\int_{M}(m+\langle\not D \psi, \psi\rangle)\left(\operatorname{div}_{g}\left(V^{\top}\right)-\left\langle V^{\perp}, \mathbf{H}\right\rangle\right) \operatorname{dvol}_{g},
\end{aligned}
$$

where $\mathbb{I}(T)=\sum_{i j} T_{i j} \mathbb{I}_{i j}^{\alpha} e_{\alpha}=-(m+\langle\not D \psi, \psi\rangle) \mathbf{H}+\left\langle\gamma\left(e_{i}\right) \not_{e_{j}} \psi, \psi\right\rangle \mathbb{I}_{i j}^{\alpha} e_{\alpha}$ denotes the contraction of $T$ and $\mathbb{I}$.

To summarize, we have

Proposition 2.1 (First variation formula) For a smooth variation $\left(u_{t}, \psi_{t}\right)$ of $(u, \psi)$ with variational field $(V, \eta)$, where each $u_{t}$ is an immersion, the first variation formula for the functional $\mathcal{A}_{S}$ is

$$
\begin{aligned}
\left.\frac{\mathrm{d}}{\mathrm{d} t}\right|_{t=0} \mathcal{A}_{S}\left(u_{t}, \psi_{t}\right)= & \int_{M} 2\langle\not D \psi, \eta\rangle-e_{i}\left\langle\eta, \gamma\left(e_{i}\right) \psi\right\rangle+\langle\mathscr{R}, V\rangle \operatorname{dvol}_{g} \\
& +\int_{M}\left\langle V^{\top}, \operatorname{div}_{g}(T)_{\sharp}\right\rangle+\left\langle V^{\perp}, \mathbb{I}(T)\right\rangle-\operatorname{div}_{g}\left(T\left(V^{\top}\right)\right) \operatorname{dvol}_{g} .
\end{aligned}
$$


Corollary 2.2 If $M$ is a closed manifold, then

$$
\left.\frac{\mathrm{d}}{\mathrm{d} t}\right|_{t=0} \mathcal{A}_{S}\left(u_{t}, \psi_{t}\right)=\int_{M} 2\langle\not D \psi, \eta\rangle+\langle\mathscr{R}, V\rangle+\left\langle V^{\top}, \operatorname{div}_{g}(T)_{\sharp}\right\rangle+\left\langle V^{\perp}, \mathbb{I}(T)\right\rangle \operatorname{dvol}_{g} .
$$

In case $M$ is complete and non-compact, if the variational field $(V, \eta)$ is compactly supported, the above formula still holds.

Some comments are in order. The above first variation formulas tell us that for a critical point $(u, \psi)$, it is necessary that $\not D \psi=0$, namely $\psi$ is a harmonic spinor along the immersion $u$. The symmetric tensor $T$ in (8) is the energy-momentum tensor for the functional $\mathcal{A}_{S}$ in the following sense: given $(u, \psi)$ where $u$ is an immersion, $g=u^{*} \bar{g}$, and given an arbitrary smooth symmetric 2-tensor $h$, let $g(t)=g+t h$ and $b_{t}, \beta_{t}$ as before, then

$$
\left.\frac{\mathrm{d}}{\mathrm{d} t}\right|_{t=0} \mathcal{A}_{S}\left(u,\left(\beta_{t} \otimes b_{t}\right) \psi ; g(t)\right)=\int_{M}-\frac{1}{2}\langle h, T\rangle \mathrm{dvol}_{g} .
$$

In particular, the diffeomorphism invariance (7) implies that

$$
\operatorname{div}_{g}(T)=0 .
$$

Now suppose $V^{\top}$ has compact support in the interior of $M$ such that it generates a local one-parameter flow, then the diffeomorphism invariance (7) together with Corollary 2.2 (where $V^{\perp}=0$ and $\eta=0$ ) implies that

$$
0=\int_{M}\left\langle\mathscr{R}+\operatorname{div}_{g}(T)_{\sharp}, V^{\top}\right\rangle \operatorname{dvol}_{g} .
$$

By decomposing the vector $\mathscr{R}$ into the tangent and normal components, i.e. $\mathscr{R}=\mathscr{R}^{\top}+\mathscr{R}^{\perp}$, we have

$$
\langle\mathscr{R}, V\rangle=\left\langle\mathscr{R}^{\top}, V^{\top}\right\rangle+\left\langle\mathscr{R}^{\perp}, V^{\perp}\right\rangle .
$$

Since $V^{\top} \in \Gamma(T M)$ can be arbitrary, we conclude that there always holds

$$
\mathscr{R}^{\top}=-\operatorname{div}_{g}(T)_{\sharp}=0 \quad \text { and hence } \quad \mathscr{R}=\mathscr{R}^{\perp} .
$$

Corollary 2.3 The variation formula for $\mathcal{A}_{S}$ on closed manifolds, or on complete manifolds with compact supported variations, has the form

$$
\left.\frac{\mathrm{d}}{\mathrm{d} t}\right|_{t=0} \mathcal{A}_{S}\left(u_{t}, \psi_{t}\right)=\int_{M} 2\langle\not D \psi, \eta\rangle+\left\langle\mathscr{R}^{\perp}+\mathbb{I}(T), V^{\perp}\right\rangle \mathrm{dvol}_{g}
$$

Proposition 2.4 The Euler-Lagrange equations for the functional $\mathcal{A}_{S}$ are ${ }^{4}$

$$
\begin{aligned}
-\mathbb{I}(T) & =\mathscr{R}, \\
\not D \psi & =0 .
\end{aligned}
$$

Solutions of (9) will be called a $S P$-minimal surface. Note that since $\psi$ is harmonic, we have

$$
\mathbb{I}(T)=-m \mathbf{H}+\left\langle\gamma\left(e_{i}\right) \not_{e_{j}} \psi, \psi\right\rangle \mathbb{I}_{i j}^{\alpha} e_{\alpha} .
$$

The regularity theory for minimal surface operator (e.g. [6]) and for Dirac operator (e.g. [12]) can be applied to the system (9), and we expect that if $M$ is a surface and $u$ is an

\footnotetext{
${ }^{4}$ It follows from the remarks above that we can replace $\mathscr{R}^{\perp}$ by $\mathscr{R}$ in the first equation.
} 
immersion of class $W^{1,2}$ while $\psi$ is of class $H^{\frac{1}{2}}$, then the solution $(u, \psi)$ is smooth. But the regularity theory is not our concern here. We will assume the solutions under consideration are smooth in the sequel, at least in lower dimensions.

\section{Relation to Dirac-harmonic Maps}

A $C^{2}$-map $u:(M, g) \rightarrow(\bar{M}, \bar{g})$ (not necessarily an immersion) is called harmonic if it is a critical point of the Dirichlet energy functional

$$
E(u)=\frac{1}{2} \int_{M}|\mathrm{~d} u|^{2} \mathrm{dvol}_{g}
$$

Let $\tau(u)=\operatorname{tr}(\nabla \mathrm{d} u)$ be the tension field of $u$, then the harmonic map equation is $\tau(u)=0$. Note that for an isometric immersion $u: M \rightarrow \bar{M}$, the tension field is the same as the mean curvature vector: $\tau(u)=\mathbf{H}$.

A pair $(u, \psi)$, where $u:(M, g) \rightarrow(\bar{M}, \bar{g})$ is a $C^{2}$ map and $\psi \in \Gamma\left(\Sigma M \otimes u^{*} T \bar{M}\right)$, is called a Dirac-harmonic map if it is a critical point of

$$
\mathcal{L}_{D}(u, \psi)=\int_{M}|\mathrm{~d} u|^{2}+\langle\not D \psi, \psi\rangle \mathrm{dvol}_{g}
$$

The metric $g$ on the domain manifold is fixed. The Euler-Lagrange equations are

$$
\begin{aligned}
2 \tau(u) & =\mathcal{R}, \\
\not D \psi & =0,
\end{aligned}
$$

where $\mathcal{R} \equiv \mathcal{R}(u, \psi)$ is defined in the same way as $\mathscr{R}$ in the previous section; namely, with respect to a local frame $\left(\epsilon_{a}\right)$ of $T \bar{M}$, we can write $\psi=\psi^{a} \otimes \epsilon_{a}$, and then with respect to a local orthonormal frame $\left(e_{i}\right)$ of $(M, g)$,

$$
\mathcal{R}(u, \psi):=\left\langle\psi^{a}, \gamma\left(e_{i}\right) \psi^{b}\right\rangle \bar{R}\left(\epsilon_{a}, \epsilon_{b}\right) \mathrm{d} u\left(e_{i}\right),
$$

see [4] for more details. The energy-momentum tensor $\mathcal{T}=\mathcal{T}_{i j} e^{i} \otimes e^{j}$ for $\mathcal{L}_{D}$ is given by

$$
\mathcal{T}_{i j}=2\left(u^{*} \bar{g}\right)_{i j}-|\mathrm{d} u|^{2} g_{i j}+\frac{1}{2}\left\langle\gamma\left(e_{i}\right) \nabla_{e_{j}} \psi+\gamma\left(e_{j}\right) \nabla_{e_{i}} \psi, \psi\right\rangle-\langle\not D \psi, \psi\rangle g_{i j}
$$

The question of interest is: suppose $(u, \psi)$ is a Dirac harmonic map, i.e. it solves (10), and suppose that the energy-momentum tensor vanishes $\mathcal{T}(u, \psi)=0$, what would the geometry specialized by $(u, \psi)$ be?

As said, if $u$ is a harmonic map from a Riemannian surface (so $m=\operatorname{dim} M=2$ ) with vanishing energy-momentum tensor, then $u$ is weakly conformal and hence a branched minimal immersion. However, in the Dirac-harmonic map case, the vanishing of the energymomentum tensor $\mathcal{T}$ does not guarantee that $u$ is a branched immersion. This prevents further investigation. But if we restrict ourselves to considering those which are immersions, we have the following

Theorem 3.1 Let $u:\left(M^{2}, g\right) \rightarrow(\bar{M}, \bar{g})$ be a smooth isometric immersion from a surface $M$ and $\psi \in \Gamma\left(\Sigma_{g} M \otimes u^{*} T \bar{M}\right)$. Suppose $\mathcal{T}(u, \psi)=0$. Then $(u, \psi)$ is a Dirac-harmonic map iff $(u, \psi)$ is an $S P$-minimal surface. 
Proof Since $u$ is an isometric immersion, $\mathrm{d} u\left(e_{i}\right)=e_{i}$, thus the $\mathscr{R}$-operator for Diracharmonic maps and the $\mathcal{R}$-operator for $S P$-minimal surfaces coincide. The energy density of $u$ is constant:

$$
|\mathrm{d} u|^{2}=\sum_{i}\left\langle\mathrm{~d} u\left(e_{i}\right), \mathrm{d} u\left(e_{i}\right)\right\rangle=\bar{g}_{i i}=m=\operatorname{dim}(M)=2 .
$$

Hence $2\left(u^{*} \bar{g}\right)_{i j}-|\mathrm{d} u|^{2} g_{i j}=0$. Now $\not D \psi=0$ together with $\mathcal{T}=0$ implies that

$$
\frac{1}{2}\left\langle\gamma\left(e_{i}\right) \nabla_{e_{j}} \psi+\gamma\left(e_{j}\right) \nabla_{e_{i}} \psi, \psi\right\rangle=0 \text {. }
$$

Therefore $T_{i j}=-2 g_{i j}$ and

$$
-\mathbb{I}(T)=2 \mathbf{H}=2 \tau(u) .
$$

The conclusion follows.

Since the functional $\mathcal{L}_{D}$ is not conformally invariant in dimensions $m \geq 3$, the energy-momentum tensor $\mathcal{T}$ cannot vanish on-shell in higher dimensions. Indeed, in dimension $m \geq 3$, if $u$ is an isometric immersion, then

$$
\operatorname{tr}(\mathcal{T})=(2-m) m+(1-m)\langle\not D \psi, \psi\rangle .
$$

If furthermore $\not D \psi=0$, then $\operatorname{tr}(\mathcal{T})=(2-m) m<0$. Note that in case $\mathcal{T}=\lambda g$, then necessarily $\lambda=2-m<0$.

Proposition 3.2 Let $u: M^{m} \rightarrow \bar{M}$ be an isometric immersion, with $m \geq 2$ and $\psi \in$ $\Gamma\left(\Sigma_{g} M \otimes u^{*} T \bar{M}\right)$ so that

$$
\mathcal{T}(u, \psi)=-(m-2) g .
$$

Then $(u, \psi)$ is a Dirac-harmonic map if and only if $(u, \widetilde{\psi})$ is an SP-minimal surface, where $\widetilde{\psi}=\sqrt{m / 2} \psi$.

The proof is similar to that of Theorem 3.1 and is omitted.

Recall that for a conformal field theory the energy-momentum tensor is always perpendicular to the metric tensor, while this fails if the action is not conformally invariant. It remains unclear to us when the two symmetric tensors $\mathcal{T}$ and $g$ would be aligned. We suspect this would also be rare and rigid.

Furthermore, notice that the $\mathscr{R}$ operator and the $\mathcal{R}$ operator has the same structure which are quadratic in the spinor part, and $\tau=\mathbf{H}$ for isometric immersions, we immediately get

Corollary 3.3 Let $u: M^{m} \rightarrow \bar{M}$ be an isometric immersion and $\psi \in \Gamma\left(\Sigma_{g} M \otimes u^{*} T \bar{M}\right)$. Let $T$ be defined as (8). If

$$
-\mathbb{I}(T)=2 \sigma \boldsymbol{H} \quad \text { for some } \sigma>0,
$$

then $(u, \psi)$ is a Dirac-harmonic map iff $(u, \widetilde{\psi}=\sqrt{\sigma} \psi)$ is an SP-minimal surface.

This includes Proposition 3.2 as a special case where $\sigma=m / 2$.

Finally, we discuss some explicit examples based on the ansatz in [15] and [1]. Consider a pair $(u, \Phi)$ where $u: M^{m} \rightarrow \bar{M}^{m+1}(c)$ is an isometrically immersed hypersurface into a space of constant sectional curvature $c$, with global unit normal $v$, and the spinor component has the form

$$
\Phi=\gamma\left(e_{j}\right) \psi \otimes e_{j}+\varphi \otimes v
$$


for some pure spinors $\psi, \varphi \in \Gamma(\Sigma M)$. The conditions for $(u, \Phi)$ to be a Dirac-harmonic map are clarified in [1, Theorem 1.1, Theorem 1.5]. Writing $H=|\mathbf{H}|$ as the mean curvature where $\mathbf{H}=\operatorname{tr}(\mathbb{I})$ (so $\mathbf{H}=H v)$, we have

$$
\not D \Phi=\left(\frac{2-m}{m} \gamma\left(e_{j}\right) \not \partial \psi-2 P_{e_{j}} \psi-\mathbb{I}_{i j} \gamma\left(e_{i}\right) \varphi\right) \otimes e_{j}+(\not \partial \varphi-H \psi) \otimes \nu,
$$

where $P$ is the Penrose operator on $\Gamma\left(\Sigma_{g} M\right)$ defined by

$$
P \psi=\nabla \psi+\frac{1}{m} \sum_{i=1}^{m} e^{i} \otimes \gamma\left(e_{i}\right) \not \psi \in \Gamma\left(T^{*} M \otimes \Sigma M\right),
$$

that is, for any $X \in \Gamma(T M)$,

$$
P_{X} \psi=\nabla_{X} \psi+\frac{1}{m} \gamma(X) \not \gamma
$$

In the case $m \geq 3$ and that $u$ is a totally umbilical immersion which is non-minimal, as in the discussion of [1], we actually have

$$
\begin{aligned}
\mathbb{I}_{i j}\left\langle\gamma\left(e_{i}\right) \not_{e_{j}} \Phi, \Phi\right\rangle= & (2-m) \mathbb{I}_{i j}\left\langle\gamma\left(e_{i}\right) \nabla_{e_{j}}^{s} \psi, \psi\right\rangle+\mathbb{I}_{i j}\left\langle\gamma\left(e_{i}\right) \nabla_{e_{j}}^{s} \varphi, \varphi\right\rangle \\
& +\mathbb{I}_{i j} \mathbb{I}_{j k}\left\langle\gamma\left(e_{i}\right) \gamma\left(e_{k}\right) \psi, \varphi\right\rangle \\
= & (2-m) \frac{H}{m}\left\langle\gamma\left(e_{i}\right) \nabla_{e_{i}}^{s} \psi, \psi\right\rangle+\frac{H}{m}\left\langle\gamma\left(e_{i}\right) \nabla_{e_{i}}^{s} \varphi, \varphi\right\rangle \\
& +2\left(\frac{H}{m}\right)^{2}\left\langle\gamma\left(e_{i}\right) \gamma\left(e_{i}\right) \psi, \varphi\right\rangle \\
= & \frac{2-m}{m} H\langle\not \gamma \psi, \psi\rangle+\frac{H}{m}\langle\not \partial \varphi, \varphi\rangle-\frac{2 H^{2}}{m}\langle\psi, \varphi\rangle .
\end{aligned}
$$

If in addition $\not D \Phi=0$, then

$$
\begin{gathered}
\frac{2-m}{m} \gamma\left(e_{j}\right) \not \partial \psi-2 P_{e_{j}} \psi-\mathbb{I}_{i j} \gamma\left(e_{i}\right) \varphi=0, \quad \forall 1 \leq j \leq m, \\
\not \varphi-H \psi=0 .
\end{gathered}
$$

From (13) we obtain

$$
(m-2) \not \partial \psi+H \varphi=0 .
$$

It follows that

$$
\mathbb{I}_{i j}\left\langle\gamma\left(e_{i}\right) \not_{e_{j}} \Phi, \Phi\right\rangle=0
$$

and hence

$$
\mathbb{I}(T)=-m \mathbf{H} .
$$

Thus in this case the Dirac-harmonic maps and the $S P$-minimal surfaces are equivalent.

In the surface case, if $\varphi=0$ in (12), then again the Dirac-harmonic maps and the $S P$ minimal surfaces are equivalent. While in the general case, whether the equivalence holds or not remains unclear. Note that when $M$ is a closed surface, there are no known examples with $\varphi \neq 0$.

Thus, on one side, we can get some examples directly from [1]; on the other hand, we cannot obtain new examples using the ansatz (12). It is also unclear whether the condition (11) is satisfied in the higher dimensional case. 
Acknowledgements The author is grateful to the referees for useful and detailed comments. He would like to thank Enno Keßler for helpful discussions. The work was done when the author was supported by Centro di Ricerca Matematica Ennio de Giorgi in Pisa.

Funding Open access funding provided by Scuola Internazionale Superiore di Studi Avanzati - SISSA within the CRUI-CARE Agreement.

Open Access This article is licensed under a Creative Commons Attribution 4.0 International License, which permits use, sharing, adaptation, distribution and reproduction in any medium or format, as long as you give appropriate credit to the original author(s) and the source, provide a link to the Creative Commons licence, and indicate if changes were made. The images or other third party material in this article are included in the article's Creative Commons licence, unless indicated otherwise in a credit line to the material. If material is not included in the article's Creative Commons licence and your intended use is not permitted by statutory regulation or exceeds the permitted use, you will need to obtain permission directly from the copyright holder. To view a copy of this licence, visit http://creativecommons.org/licenses/by/4.0/.

\section{References}

1. Ammann, B., Ginoux, N.: Some examples of Dirac-harmonic maps. Lett. Math. Phys. 109, 1205-1218 (2019)

2. Bär, C., Gauduchon, P., Moroianu, A.: Generalized cylinders in semi-Riemannian and spin geomtry. Math. Z. 249, 545-580 (2005)

3. Bourguignon, J.-P., Gauduchon, P.: Spineurs, opérateurs de Dirac et variations de métriques. Commun. Math. Phys. 144, 581-599 (1992)

4. Chen, Q., Jost, J., Li, J., Wang, G.: Dirac-harmonic maps. Math. Z. 254, 409-432 (2006)

5. Deligne, P. et al (eds): Quantum Fields and Strings: A Course for Mathematicians. American Mathematical Society, Providence, RI (1999)

6. Giaquinta, M., Martinazzi, L.: An Introduction to the Regularity Theory for Elliptic Systems, Harmonic Maps and Minimal Graphs. Lecture Notes (Scuola Normale Superiore), vol. 11. Edizioni della Normale (2012)

7. Ginoux, N.: The Dirac Spectrum. Lecture Notes in Mathematics, vol. 1976. Springer, Berlin, Heidelberg (2009)

8. Jost, J.: Minimal surfaces and Teichmüller theory. In: Yau, S.-T. (ed.) Tsinghua Lecture Notes on Geometry and Analysis, pp. 149-211. International Press, Boston (1997)

9. Jost, J.: Geometry and Physics. Springer, Berlin (2009)

10. Jost, J.: Riemannian Geometry and Geometric Analysis, 6th edn. Universitext. Springer, Heidelberg (2011)

11. Jost, J.: Compact Riemann Surfaces, 3rd edn. Universitext. Springer, Berlin (2013)

12. Jost, J., Keßler, E., Tolksdorf, J., Wu, R., Zhu, M.: Regularity of solutions of the nonlinear sigma model with gravitino. Commun. Math. Phys. 358, 171-197 (2018)

13. Jost, J., Keßler, E., Tolksdorf, J., Wu, R., Zhu, M.: Symmetries and conservation laws of a nonlinear sigma model with gravitino. J. Geom. Phys. 128, 185-198 (2018)

14. Jost, J., Keßler, E., Tolksdorf, J., Wu, R., Zhu, M.: From harmonic maps to the nonlinear supersymmetric sigma model of quantum field theory:, At the interface of theoretical physics, Riemannian geometry and nonlinear analysis. Vietnam. J. Math. 47(1), 39-67 (2019)

15. Jost, J., Mo, X., Zhu, M.: Some explicit constructions of Dirac-harmonic maps. J. Geom. Phys. 59, 15121527 (2009)

16. Jost, J., Wu, R., Zhu, M.: Energy quantization for a nonlinear sigma model with critical gravitinos. Trans. Amer. Math. Soc. Ser. B 6, 215-244 (2019)

17. Keßler, E.: Supergeometry, Super Riemann Surfaces and the Superconformal Action Functional. Lecture Notes in Mathematics, vol. 2230. Springer, Cham (2019)

18. Blaine Lawson Jr., H., Michelsohn, M.-L.: Spin Geometry. Princeton University Press, Princeton, NJ (1989)

19. Maier, S.: Generic metrics and connections on spin- and $\operatorname{spin}^{c}$-manifolds. Commun. Math. Phys. 188, 407-437 (1997)

20. Polchinski, J.: String Theory: an Introduction to Bosonic String. Cambridge Monographs on Mathematical Physics. Cambridge University Press, Cambridge (1998) 
21. Sacks, J., Uhlenbeck, K.: The existence of minimal immersion of 2-spheres. Ann. Math. (2) 113, 1-24 (1981)

22. Tromba, A.J.: Teichmüller Theory in Riemannian Geometry. Birkhäuser, Basel (2012)

23. Wu, R.: Analysis of a Two-Dimensional Nonlinear Sigma Model with Gravitino. PhD Thesis, Universität Leipzig (2017)

Publisher's Note Springer Nature remains neutral with regard to jurisdictional claims in published maps and institutional affiliations. 